\title{
Identification and subcellular localisation of hexokinase-2 in Nosema bombycis
}

\author{
Jiancheng Sun ${ }^{1 *}$, Feng Zhu ${ }^{2,4^{*}}$, Hongli Chen ${ }^{1}$, Mingshuai Yao ${ }^{1}$, Guanyu Zhu ${ }^{1}$, Yiling Zhang ${ }^{1,3}$, Qiang Wang ${ }^{1,3}$ and \\ Zhongyuan Shen ${ }^{1,3}$
}

\author{
${ }^{1}$ Jiangsu University of Science and Technology, Zhenjiang, Jiangsu Province, China; \\ ${ }^{2}$ College of Life Sciences, Zaozhuang University, Zaozhuang, Shandong Province, China; \\ ${ }^{3}$ Sericultural Research Institute, Chinese Academy of Agricultural Sciences, Zhenjiang, Jiangsu Province, China; \\ ${ }^{4}$ Institute of Sericulture and Apiculture, Yunnan Academy of Agricultural Sciences, Mengzi, Yunnan Province, China \\ *these authors contributed equally
}

\begin{abstract}
Hexokinase (HXK) is the first key enzyme in the glycolytic pathway and plays an extremely important role in energy metabolism. By searching the microsporidian database, we found a sequence (NBO_27g0008) of Nosema bombycis Nägali, 1857 with high similarity to hexokinase-2, and named it as NbHXK2. The NbHXK2 gene has 894 bp and encodes 297 amino acids with 34.241 $\mathrm{kD}$ molecular weight and 5.26 isoelectric point. NbHXK2 contains 31 phosphorylation sites and 4 potential $\mathrm{N}$-glycosylation sites with signal peptides and no transmembrane domain. Multiple sequence alignment showed that NbHXK2 shares more than $40 \%$ amino acid identity with that of other microsporidia, and the homology with hexokinase-2 of Nosema tyriae Canning, Curry, Cheney, Lafranchi-Tristem, Kawakami, Hatakeyama, Iwano et Ishihara, 1999, Nosema pyrausta (Paillot, 1927) and Nosema ceranae Fries, Feng, da Silva, Slemenda et Pieniazek, 1996 was $89.17 \%, 87.82 \%$ and $69.86 \%$, respectively. Phylogenetic analysis based on the amino acid sequence of hexokinase showed that all microsporidia cluster together in the same clade, and are far away from animals, plants and fungi, and that $N$. bombycis is closely related to N. tyriae; N. pyrausta; $N$. ceranae and Nosema apis Zander, 1909. Immunolocalisation with the prepared polyclonal antibody showed that NbHXK2 was mainly distributed in the cytoplasm and plasmalemma in proliferative, sporulation stage and mature spore of $N$. bombycis. qRT-PCR assay showed that the $N b H X K 2$ expressed at higher level during spore germination and at early stage of proliferation. These results indicate that $N$. bombycis may use its own glycolytic pathways to supply energy for infection and development, especially germination and in the early stage of proliferation, and acquire energy from the host through certain ways as well.
\end{abstract}

Keywords: microsporidia, silkworm, glucose metabolism, NbHXK2

Microsporidia are obligate intracellular parasites that have broad host range including invertebrates and vertebrates. About 1,500 species of microsporidia have been found in 187 genera (Franzen 2005, Vávra and Lukeš 2013). The first microsporidium, to Nosema bombycis Nägali, 1857 was discovered in the silkworm by Nägeli in 1857 (Wittner and Weiss 1999). Pebrine disease is one of the most devastating diseases in sericulture production. The outbreak of the disease has caused huge economic losses to the sericulture industry. Therefore, pebrine disease is listed as the only quarantine object for sericulture production all over the world (Fu et al. 2016).

Hexokinase (HXK) catalyses the phosphorylation of glucose to form 6-phosphate-glucose (G-6-P) and plays a vital role in energy metabolism (Wilson 1995, Cárdenas et al. 1998). Hexokinases have been identified in a variety of species that range from bacteria, yeast and plants to vertebrates including humans. Multicellular organisms such as plants and animals often have more than one hexokinase isoform. For example, four hexokinase isozymes have been identified in mammalian species according to their electrophoretic mobility.

The hexokinases I, II, III isozymes are $100 \mathrm{kDa}$ molecules thought to have evolved by duplication and fusion of a gene encoding an ancestral $50 \mathrm{kDa}$ hexokinase. Thus, these isozymes display internal sequence repetition, and the $\mathrm{N}$ - and C-terminal halves have extensive sequence similarity both to each other and to other members of the hexokinase family.

Hexokinases IV isozyme, commonly known as glucokinase, is about $50 \mathrm{kDa}$ in size (Wilson 2003, Li et al. 2014). Moreover, recent studies have found that HXK is involved 
in the interaction between pathogens and hosts. In macrophages, HXK is an innate immune receptor for the detection of bacterial peptidoglycans (Wolf et al. 2016).

Although microsporidia display extreme degree of genetic reduction resulting in incomplete metabolism that is particularly evident in ATP-generating pathways (Wiredu Boakye et al. 2017), the microsporidial genomes can expand through several common molecular mechanisms such as gene duplication, horizontal gene transfer and transposable element expansion (Pan et al. 2013).

Data from two microsporidia species of Nematocida Troemel, Félix, Whiteman, Barrière et Ausubel, 2008 revealed that the use of a large number of rapidly evolving species-specific proteins represents a common strategy for microsporidia to interact with their hosts (Reinke et al. 2017). Microsporidia can acquire transporters that import nucleosides to fuel rapid growth. In addition, microsporidian hexokinases may be targeted into the host cell to reprogram it toward biosynthesis (Cuomo et al. 2012).

Research on hexokinases from microsporidia N. bombycis and Nosema ceranae Fries, Feng, da Silva, Slemenda et Pieniazek, 1996 demonstrated the capability of microsporidia-secreted HKs to phosphorylate glucose in infected cells, suggesting that they actively mediate the effects of the parasite on host metabolism (Dolgikh et al. 2019). Nosema bombycis has two hexokinase isoforms. In this study, we identified one hexokinase isoform (EOB14451.1) of $N$. bombycis from which we infer that this hexokinase may supply energy for infection and early development by both its own glycolytic pathways and the host.

\section{MATERIALS AND METHODS}

\section{Materials}

Nosema bombycis strain was preserved in the Laboratory of Silkworm Physiology and Pathology, Sericulture Research Institute of the Chinese Academy of Agricultural Sciences, Zhenjiang, China. The BmN cell line was maintained at $27^{\circ} \mathrm{C}$ in TC-100 insect medium supplemented with $10 \%(\mathrm{v} / \mathrm{v})$ fetal bovine serum.

\section{Bioinformatic analysis}

By searching the microsporidian database (http://microsporidiadb.org/), we found a sequence in the microsporidian $N$. bombycis, namely NbHXK2 (NBO_27g0008, Accession No. EOB14451.1), with high similarity to hexokinase-2. The isoelectric point and molecular weight of $\mathrm{NbHXK} 2$ were predicted using ExPaSy proteomic tools (http://web.expasy.org/compute_pi/).

The signal peptide and the transmembrane domain were predicted using ANTHEPRO 6.3.6 software and TMHMM Server v.2.0 (http://www.cbs.dtu.dk/services/TMHMM/). Subcellular localisation was predicted using Cell-PLoc 2.0 (http://www. csbio.sjtu.edu.cn/bioinf/Cell-PLoc-2/). The N-terminal region of NbHXK2 was used to blast homologous sequences in NCBI (https://www.ncbi.nlm.nih.gov/). The homologous sequences were compared using DNAMAN. The phylogenetic tree was constructed by the NJ method using MEGA6.

\section{Gene cloning, recombinant protein expression and purification}

Folia Parasitologica 2020, 67: 023
In this study, NbHXK2 genomic DNA was extracted from $N$. bombycis as previously described (Fredricks et al. 2005). The $\mathrm{NbHXK} 2$ was amplified by PCR amplification reaction: $96^{\circ} \mathrm{C}$ for $6 \min ; 95^{\circ} \mathrm{C}$ for $20 \mathrm{~s}, 44^{\circ} \mathrm{C}$ for $15 \mathrm{~s}$, and $72^{\circ} \mathrm{C}$ for $1 \mathrm{~min}, 35$ cycles; and $72^{\circ} \mathrm{C}$ for $10 \mathrm{~min}$ using the forward primer $5^{\prime}$ - CGGGATCCATGATTGATTACGTATT -3' containing an BamH I restriction site, and the reverse primer 5'-GCGTCGACTTAATATATAATTCGATG-3' containing a Sal I restriction site. The PCR products were separated on a $1 \%$ agarose gel, purified with the AXY Prep DNA fragment purification kit (Corning, Shanghai, China) and cloned into the pMD19-T vector (TaKaRa, Dalian, China).

Then, the vector was transformed into Escherichia coli strain Top10 competent cells. Positive clones were confirmed by bluewhite selection and the isolated plasmids were sequenced by Sangon BioTech (Shanghai, China).

Then, the pMD19-T-NbHXK2 recombinant plasmid and the pET28a expression vector were digested with BamH I and Sal I, ligated and transformed into Top10 cells. Positive recombinant plasmids were confirmed by enzyme digestion and transformed into $E$. coli BL21 (DE3) cells. These bacterial cells were induced for $6 \mathrm{~h}$ at $37^{\circ} \mathrm{C}$ with $0.6 \mathrm{mM}$ IPTG.

After induction, bacterial cells were lysed in pre-cooled PBS buffer ( $\mathrm{pH}$ 7.4) using supersonic waves, ultrasonic supernatant and precipitation were collected respectively by centrifugation at $12000 \mathrm{rpm}$ for $10 \mathrm{~min}$. Ultrasonic precipitation was dissolved overnight in a binding buffer (containing 8M urea), and ultrasonic supernatant and precipitation were boiled for $10 \mathrm{~min}$ in SDS extraction buffer. The recombinant protein pET-28a- NbHXK2 was identified by SDS-PAGE and mass spectrometry (Sangon BioTech). The expressed recombinant proteins were purified with the His-Bind purification kit (GE Healthcare, Uppsala, Sweden) according to the manufacturer's instructions.

\section{Preparation of polyclonal antibodies and immunoblotting}

Preparation of polyclonal antibodies and immunoblotting protocols to detect the HXK-1 protein of N. bombycis were performed as previously described (Wu et al. 2008, Li et al. 2009). Briefly, each rabbit (New Zealand) was subcutaneously injected every 14 days with the NbHXK2 recombinant protein mixed with Freund's adjuvant (Sigma) for four times. Rabbits in the negative control group were injected with PBS. Ten days after the last injection, serum was collected from rabbit blood and purified.

\section{Subcellular localisation of NbHXK2 protein in Nosema bombycis}

$\mathrm{BmN}$ cells were inoculated with and grown on coverslips for 24 h. Proliferating N. bombycis in the host cells were collected by disruption of the infected $\mathrm{BmN}$ cells followed by filtration through glass wool, then fixed on glass slides (Taupin et al. 2006, Tsaousis et al. 2008).

Proliferative cells obtained, as described above, mature spores, germinated spores of $N$. bombycis, and healthy Bmcells were fixed with $4 \%$ paraformaldehyde and permeabilised with $1 \%$ Triton X-100 for $1 \mathrm{~h}$ at room temperature, respectively, then blocked with a blocking regent comprising of $0.5 \%(\mathrm{w} / \mathrm{v}) \mathrm{BSA}$ and $10 \%(\mathrm{w} / \mathrm{v})$ goat serum for $1 \mathrm{~h}$, and incubated with $1: 200$ dilutions of anti-NbHXK2 serum at $37^{\circ} \mathrm{C}$ for $1 \mathrm{~h}$. After washing with PBS three times (10 min each time), Alexa Fluor 488-conjugated goat anti-rabbit IgG (Sangon BioTech) was used to detect the binding of primary antibodies at room temperature. The nuclei were stained with 4, 6-diamidino-2-phe- 
nylindole (DAPI) (Sangon BioTech, Shanghai, China) for $40 \mathrm{~min}$. Samples were observed and imaged with Olympus TH4-200 fluorescence microscope and Olympus DP80 camera.

Analysis of transcriptional activity of NbHXK2 of Nosema bombycis

The fifth instars of silkworm were fed on mulberry leaves which had been already smeared with $2 \times 10^{8} \mathrm{ml}^{-1}$ of $N$. bombycis suspension. The silkworms were dissected to obtain the mid-gut which was washed with PBS at 10 different post-infected time (2, $6,12,24,48,72,96,120,144$ and 168 hours), and preserved at $-80^{\circ} \mathrm{C}$. Then, the total RNA of the silkworm mid-gut was extracted according to the TaKaRa MiniBEST Universal RNA Extraction Kit instructions, and stored at $-80^{\circ} \mathrm{C}$. The reverse transcription reaction solution was prepared according to the instructions of the PrimeScriptTM RT Master Mix (Perfect Real Time), and the reaction mixture was mixed and incubated at $37^{\circ} \mathrm{C}$ for 30 $\mathrm{min}$, then $85^{\circ} \mathrm{C}$ for $5 \mathrm{~s}$. After reaction, cDNA was stored at $-20^{\circ} \mathrm{C}$. The qPCR assay was performed on an Applied Biosystems 7300 Fast Real Time PCR System according to the qPCR kit SYBR ${ }^{\circledR}$ Premix Ex TaqTM II (Tli RNaseH Plus) instructions (TaKaRa). The specific primers NbHXK2-qF: 5'-TTATAGCCGCCTTAACATTGG-3', NbHXK2-qR: 5'-TTGGGTTATCAAATCCAGATCC-3' were used for qPCR reaction. The $\beta$ - tubulin gene of $N$. bombycis served as the reference gene. The relative gene expression was calculated using delta-delta $\mathrm{Ct}$.

\section{RESULTS}

\section{Cloning and characterisation of $\mathrm{NbHXK} 2$ gene}

Sequencing results showed that the $N b H X K 2$ gene contains a complete ORF of 894 bp in length and encodes 297 amino acids. It is predicted that the NbHXK2 is $34.241 \mathrm{kD}$ with an isoelectric point of 5.26, and contains 31 phosphorylation sites and 4 potential N-glycosylation sites without transmembrane domain, and has two signal peptides (25 aa-27 aa, 115 aa-117 aa). The NbHXK2 retains only two functional residues active site Lys176 and Ser419 conserved in the Saccharomyces cerevisiae (Meyen 1838) HXK1 and Trachipleistophora hominis Hollister, Canning, Weidner, Field, Kench et Marriott, 1996 isoforms HK2 and 3 (Wiredu Boakye et al. 2017).

The secondary structure of the protein mainly consists of $\alpha$-helix (54.55\%), extended fragment (17.80\%) and random coil (27.61\%). Multiple sequence alignment showed that NbHXK2 shares more than $40 \%$ amino acid identity with hexokinases of most microsporidia such as Encephalitozoon hellem Didier, Didier, Friedberg, Stenson, Orenstein, Yee, Tio, Davis, Vossbrinck, Millichamp et Shadduck, 1991; Encephalitozoon romaleae Lange, Johny, Baker, Whitman et Solter, 2009; Encephalitozoon intestinalis (Cali, Kotler et Orenstein, 1993); Encephalitozoon cuniculi Levaditi, Nicolau et Schoen, 1923; T. hominis and Nosema apis Zander, 1909, and that the homology with analogue of Nosema tyriae Canning, Curry, Cheney, Lafranchi-Tristem, Kawakami, Hatakeyama, Iwano et Ishihara, 1999; Nosema pyrausta (Paillot, 1927), and Nosema ceranae was $89.17 \%, 87.82 \%$, and $69.86 \%$, respectively (Fig.1).
Phylogenetic analysis showed that all the microsporidia clustered together in the same clade, which was far away from Homo sapiens, Solanum tuberosum, Drosophila melanogaster, Bombyx mori and fungi such as Basidiomycota, Ascomycota, Chytridiomycota, Glomeromycota, Zygomycota, suggesting that the microsporidia share the close evolutionary relationship (Fig. 2).

\section{NbHXK2 recombinant protein expression and specificity of polyclonal antibody}

The NbHXK2 recombinant protein was expressed as inclusion bodies in Escherichia coli BL21 (DE3). Mass spectrometry result showed that the sequence similarity of the NbHXK2 recombinant protein is consistent with that of HXK2 in the Nosema bombycis. The NbHXK2 recombinant protein was purified by nickel column affinity chromatography, and the purified NbHXK2 recombinant protein was obtained by imidazole elution with different concentration gradients (see Fig. S1 in the Supplementary material). The concentration of $\mathrm{NbHXK} 2$ recombinant protein was $0.7 \mathrm{mg} / \mathrm{mL}$ by BCA method.

The specificity of the polyclonal antibody was tested by Western blotting. A protein of approximately $35 \mathrm{kDa}$ was detected in the total protein extracted from N. bombycis and NbHXK2 recombinant protein while the NbHXK2 protein polyclonal antibody served as a primary antibody and HRP-labeled goat anti-rabbit antibody as a secondary antibody (Fig. 3), whereas no band was detected when the pre-immune serum was used as control. The size is consistent with the predicted molecular weight of the $\mathrm{NbHXK} 2$ protein.

\section{Subcellular localisation of NbHXK2}

The distribution of NbHXK2 in $N$. bombycis at different developmental stages was observed by immunofluorescence microscopy. In all experiments, pre-immune serum of rabbits was used as negative control, and no green fluorescence signal was observed in $N$. bombycis (Fig. 4). In the mature spores, the green fluorescence was mainly distributed in the cytoplasm of spore and plasma membrane (Fig. 4A, Fig. $\mathrm{S} 2 \mathrm{~A}$ ), and no fluorescence was observed on the nucleus.

After the spores germination, strong green fluorescent signal is clearly visible inside the polar tube which were extruded to outside of the spore, and there is weak green fluorescence at the periphery underlying the spore wall (Fig. 4B, Fig. S2B), indicating that most of the NbHXK2 protein is ejected outside the spore together with the sporoplasm and a little of protein was residual on the site where the plasma membrane originally located. During proliferative stage, the green fluorescent signal mainly appears in the interior and near the plasma membrane of the replicating cells. No fluorescence was observed in the cytoplasm of the BmN cells, indicating that NbHXK2 was not secreted into the host cells during proliferation of $N$. bombycis (Fig. 4C, Fig. S2C).

When $N$. bombycis developed to the sporulation stage, the green fluorescence was mainly concentrated around the plasma membrane (Fig. 4D, Fig. S2D). As N. bombycis progressed to maturation, many pear-shaped immature spores and oval mature spores have been released from 


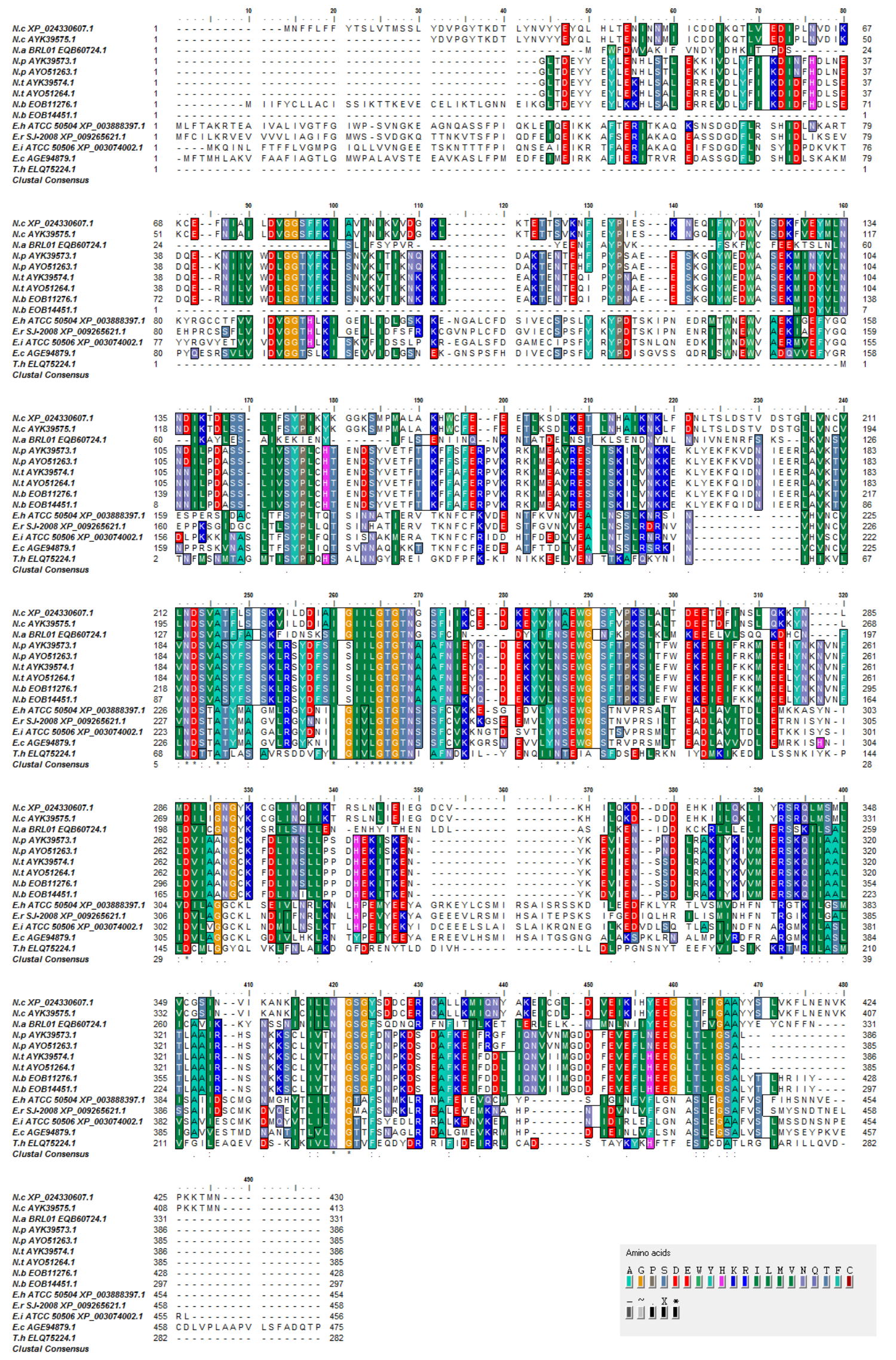

Fig. 1. Amino acid alignment of hexokinases from Nosema bombycis Nägali, 1857 and other microsporidian species $(\mathrm{p}<0.05)$. 


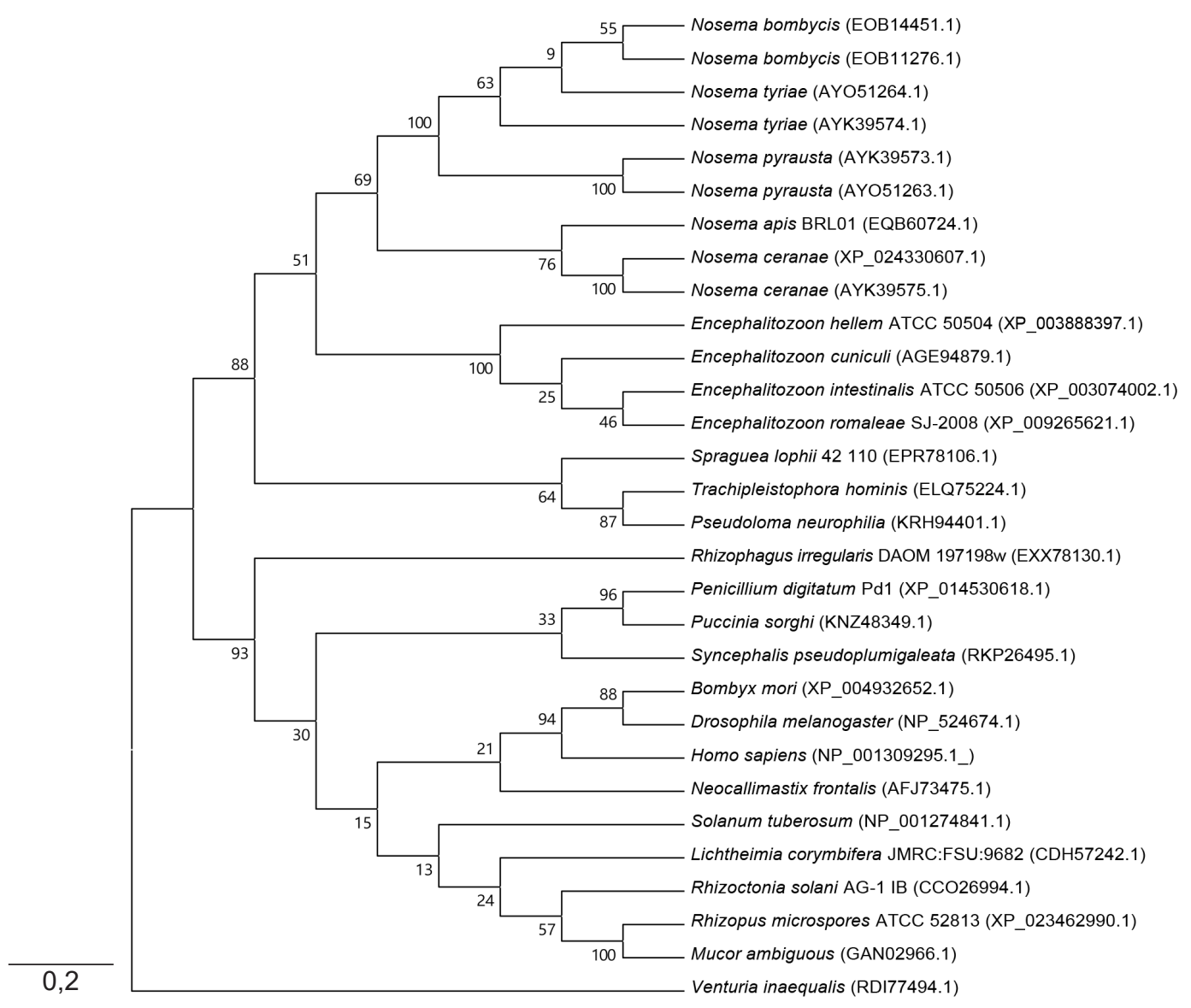

Fig. 2. Phylogenetic tree of hexokinase from microsporidian species and animal, plant and fungus. The maximum likelihood (ML) tree was constructed using MEGA 6.0 software and bootstrap analyses were performed employing JTT model-based distance matrices generated from 1000 resamplings of the alignments. Labels on branches indicate bootstrap values in percentages.

A

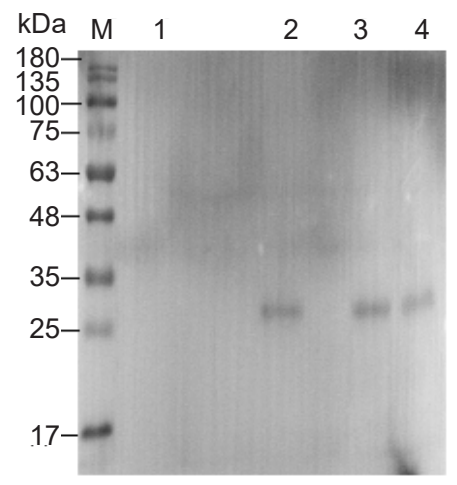

B

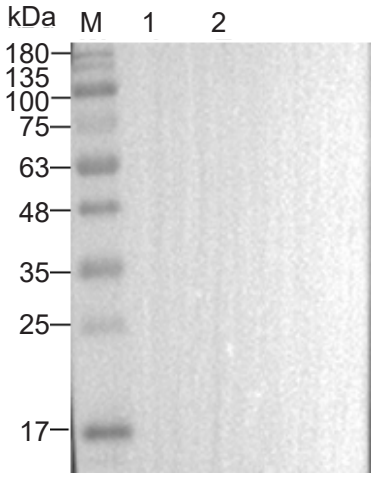

Fig. 3. Western blot analysis of polyclonal antibody against NbHXK2 recombinant protein. A - polyclonal antibody against NbHXK2. M - Protein molecular marker 1 - PBS 2 - NbHXK2 recombinant protein 3,4 - Total protein of Nosema bombycis Nägali, 1857; B - pre-immune serum; M - Protein molecular marker 1 - NbHXK2 recombinant protein 2 - Total protein of $N$. bombycis

the dissociated $\mathrm{BmN}$ cells, strong green fluorescence appeared throughout the spores (Fig. 4E), especially the plasma membrane (Fig. S2E). The above results indicate that NbHXK2 protein was expressed through all life stages and is mainly distributed in the cytoplasm and plasma membrane of $N$. bombycis.

\section{Transcriptional profile of NbHXK2 in Nosema bombycis infected midgut of silkworm}

The transcription level of the $N b H X K 2$ gene at different post-infection time was detected by qPCR with $\beta$-tubulin of $N$. bombycis as the reference gene. The relative expression level was higher in the early stage of infection (2-12 h), and decreased significantly from $24 \mathrm{~h}$ to $72 \mathrm{~h}$. It is slightly increased at $96 \mathrm{~h}$ and then decreased rapidly at $120 \mathrm{~h}$, and kept the lowest at $144 \mathrm{~h}$ (Fig. 5). The results suggest that NbHXK2 is related to the development of $N$. bombycis, especially involved in the energy metabolism of infection, early proliferation and sporulation phase of $N$. bombycis.

\section{DISCUSSION}

Hexokinase is widely present in many species and is the rate-limiting enzyme in the glycolytic pathway. We searched for the hexokinase protein in the databases, and found that the hexokinases existed commonly in the fungal kingdom, such as Basidiomycota, Ascomycota, Glomeromycota, Chytridiomycota, and Zygomycota. In the two basal fungi Chytridiomycota and Zygomycota, we found that the proteins annotated as hexokinase are present in many species of the Zygomycota, such as Mucor ambiguous, Syncephalis pseudoplumigaleata, Rhizopus microsporus, etc., and only one species, Neocallimastix frontalis, in the Chytridiomycota. 


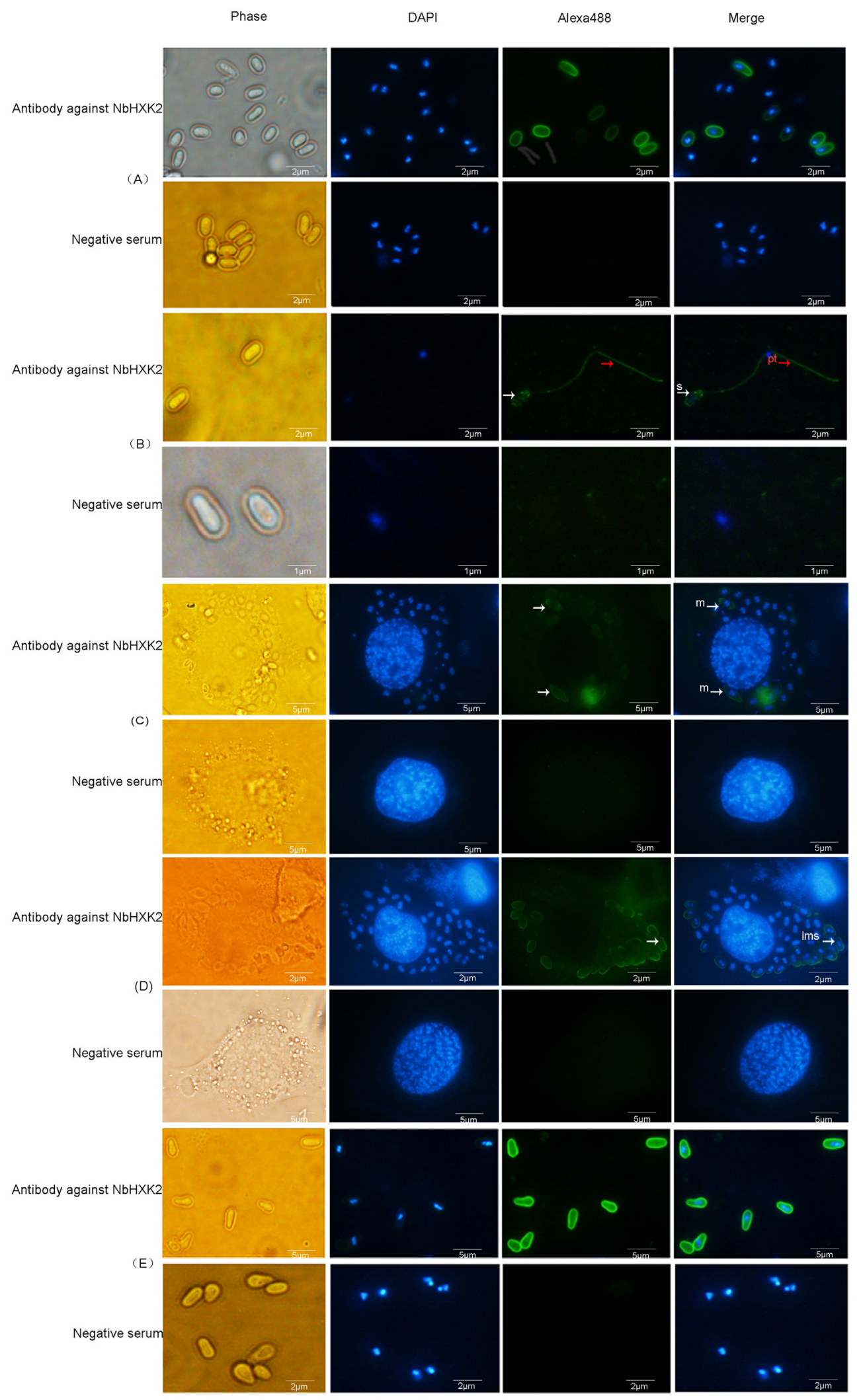

Fig. 4. Subcellular localisation of hexokinase-2 in Nosema bombycis Nägali, 1857. A - mature spores; B - germinating spores; red arrow indicates the polar tube in which the sporoplasm is releasing from the spore; white arrow represents the germinated spore shell; $\mathbf{C}$ - proliferative cell; white arrow indicates meront (dividing nuclei) in the $\mathrm{BmN}$ cell; $\mathbf{D}$ - during sporoblasts and immature spores; white arrow represents the pear-shaped immature spores; $\mathbf{E}$ - spore maturation. Abbreviations: $\mathrm{pt}$ - polar tube, $\mathrm{s}-\mathrm{spore}, \mathrm{m}-\mathrm{meront}$, ims - immature spore. 


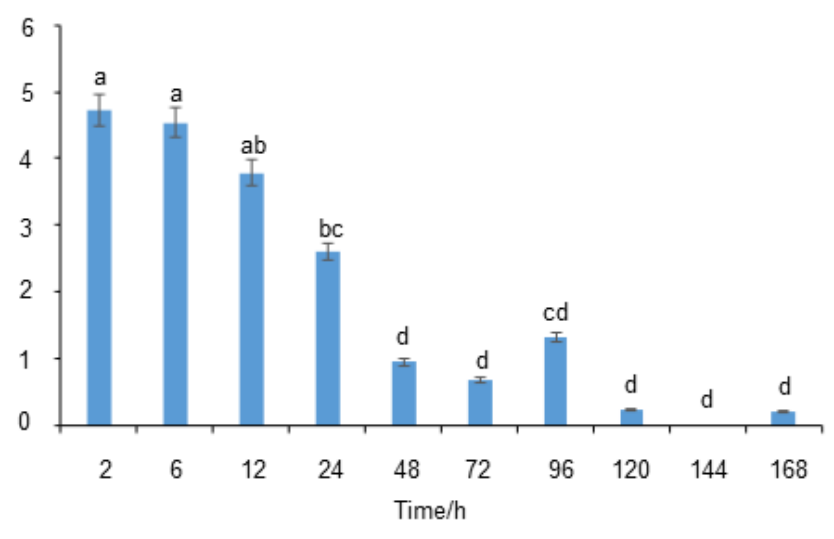

Fig. 5. The relative expression level of NbHXK2 at different post-infection time. Error bars represent the standard deviations of three independent replicates.

Among the microsporidia, we found that the hexokinase existed in the genus Nosema (N. bombycis, N. tyriae; N. pyrausta; N. ceranae and N. apis) and Encephalitozoon (E. intestinalis; E. cuniculi and E. hellem), as well as Spraguea lophii Vávra et Sprague, 1976; Trachipleistophora hominis; Pseudoloma neurophilia Matthews, Brown, Larison, Bishop-Stewart, Rogers et Kent, 2001; etc. The amino acid sequence alignment showed that $N$. bombycis has higher homology with $N$. tyriae; $N$. pyrausta and $N$. ceranae, and lower homology with other genera in microsporidia.

Phylogenetic analysis showed that all the microsporidia clustered together in the same clade, especially species of Nosema, and far away from animals (Homo sapiens; Drosophila melanogaster; Bombyx mori), plant (Solanum tuberosum) and fungi such as Rhizoctonia solani Kühn, 1858; Puccinia sorghi Schweinitz, 1834; Venturia inaequalis (Cooke, 1866); Penicillium digitatum; Rhizophagus irregularis; Neocallimastix frontalis (Braune, 1913); Mucor ambiguous; Syncephalis pseudoplumigaleata pseudoplumigaleata Benny et Ho, 2017 and Rhizopus microsporus, which indicated that the genetic relationship between microsporidia is closer.

Obligate intracellular parasites can steal energy from host cells in a variety of ways. Rickettsia and chlamydia acquire energy molecules such as ATP directly from host cells via ATP/ADP transporters (Krause et al. 1985, Trentmann et al. 2007). Encephalitozoon cuniculi contains four copies of the ATP/ADP transporter of which three are located in the cell membrane of E. cuniculi, and are involved in the transport of the host cell cytoplasmic ATP. The other is located in the mitosome of E. cuniculi and provide ATP for the metabolism of mitosome (Tsaousis et al. 2008).

An infiltrative cell coat, termed plaque matrix (PQM), which lies at the interface between the microsporidian T. hominis and the mammalian host cytoplasm, has been identified. The presence of two secreted HK isoforms implicates that the PQM theft from the host cell in orchestrating metabolic energy (Ferguson and Lucocq 2018). In addition, a series of nucleotide transporters (NTTs) has been found in microsporidia and steal energy molecules such as ATP from host cells (Heinz et al. 2014, Dean et al. 2016).
Bioinformatic analysis predicted that the NbHXK2 has signal peptide, which implies that NbHXK2 may be a secreted protein. However, our study found that this protein is mainly distributed in the cytoplasm and the plasma membrane of $N$. bombycis, and was not secreted into the host cell during infection and intracellular parasitism. Actually, the hexokinase in $N$. bombycis has two isoforms; the other isoform (Accession No. EOB11276.1) can be secreted into the host cell as a secreted protein (Huang et al. 2018) and phosphorylate glucose in infected cells (Dolgikh et al. 2019), indicating that $N$. bombycis can acquire energy from the host.

In this study, we found that $\mathrm{NbHXK2}$ was expressed relatively at high level at the infection and early proliferative stage (2-12 hpi) of $N$. bombycis, which is consistent with the transcriptional profile of the Nematocida hexokinase, which was highly expressed at 8 hpi (Cuomo et al. 2012). The NbHXK2 was distributed in the cytoplasm of dormant spore. When the spore was undergoing germination, most of the NbHXK2 released through the everted polar tube (Fig. 4A,B), indicating that accompanying by germination of the dormant spore the NbHXK2 was probably entered into the host cell together with sporoplasm, which will accomplish proliferation in the host cell.

During the early proliferative stage, at which the cell of $N$. bombycis is fusiform in shape and contains multi-nuclei, the NbHXK2 was mainly distributed in inner periphery, especially the two ends, of the meront (Fig. 4C). In the sporoganic phase, the NbHXK2 protein was further concentrated in the plasma membrane of sporoblast. Moreover, this phenomenon appeared more significantly in immature spores whose nuclei become more dense and smaller (Fig. 4D). As the functional active site of hexokinase, Lys176, Asp211, Thr234, Ser 419, Glu269 and Glu302 play important roles in ATP binding, glucose binding and catalytic bases in S. cerevisiae (HXK1, HXK2) and T. hominis (HK2, HK3) (Wiredu Boakye et al. 2017). However, NbHXK2 only contains two conserved functional active sites, Lys176 and Ser419 (ATP binding), which may imply that the NbHXK2 has lost some functions but retains the ability to bind ATP.

Trachipleistophora hominis forms sporophorous vacuole at the late developmental stage (Ferguson and Lucocq 2018), whereas $N$. bombycis interacts extensively with the host cell throughout the parasite life cycle until mature spores formed. Although we have not found distinct evidence that the NbHXK2 has been secreted into the host cell, this protein was clearly gathering to the plasma membrane during proliferative phase and sporulation phase. These results suggest that $N$. bombycis may provide energy by its own glycolytic pathway, especially during the infection and early proliferative stage at which $N$. bombycis has not established close relationship with the host cell, and that $N$. bombycis may acquire ATP from host through infiltrating its hexokinase into the host cell as well.

Microsporidia possess genes involved in metabolic processes such as nitrogen metabolism, transport, stress, biosynthesis, cellular metabolic processes, and biological regulation to complete basic metabolism. Otherwise, as 
an obligate intracellular parasitic single-cell eukaryotes, microsporidia display a variable reduction in glycolytic pathways, which is combined with significant loss of mitochondrial functions including oxidative phosphorylation. They are obliged to acquire nutrition from the host for its rapid replication and differentiation within the host cells, which may be the best evidence for long-term co-evolution of parasite and host.
Acknowledgements. This work was supported by the earmarked fund for the China Agriculture Research System and Postgraduate Research \& Practice Innovation Program of Jiangsu Province (KYCX19_1719). We are also grateful to all who provided the means for us to access free software, which we have used and cited in this article. We would also like to thank all partners and laboratory staff for kindly help and criticism.

\section{REFERENCES}

Cárdenas M.L., Cornish-Bowden A., Ureta T. 1998: Evolution and regulatory role of the hexokinases. Biochim. Biophys. Acta 1401: 242-264.

Cuomo C.A., Desjardins C.A., Bakowski M.A., Goldberg J., Ma A.T., Becnel J.J., Didier E.S., Fan L., Heiman D.I., Levin J.Z., Young S., Zeng Q., Troemel E.R. 2012: Microsporidian genome analysis reveals evolutionary strategies for obligate intracellular growth. Genome Res. 22: 2478-2488.

Dean P., Hirt R.P., Embley T.M. 2016: Microsporidia: why make nucleotides if you can steal them? PLoS Pathogens 12: e1005870.

Dolgikh V.V., Tsarev A.A., Timofeev S.A., Zhuravlyov V.S. 2019: Heterologous overexpression of active hexokinases from microsporidia Nosema bombycis and Nosema ceranae confirms their ability to phosphorylate host glucose. Parasitol. Res. 118 1511-1518.

Ferguson S., Lucoce J. 2018: The invasive cell coat at the microsporidian Trachipleistophora hominis-host cell interface contains secreted hexokinases. Microbiologyopen 8: e00696.

Franzen C. 2005: How do microsporidia invade cells? Folia Parasitol. 52: $36-40$.

Fredricks D., Smith C., Meier A. 2005: Comparison of six DNA extraction methods for recovery of fungal DNA as assessed by quantitative PCR. J. Clin. Microbiol. 43: 5122-5128.

Fu Z.W.K., He X.K., Cai S.F., LiU H., He X.Y., Li M.Q., Lu X.M. 2016: Quantitative PCR for detection of Nosema bombycis in single silkworm eggs and newly hatched larvae. J. Microbiol. Methods. 120: 72-78.

Heinz E., Hacker C., Dean P., Mifsud J., Goldberg A.V., Williams T.A., Nakjang S., Gregory A., Hirt R.P., LuCOCQ J.M., Kunji E.R., Embley T.M. 2014: Plasma membrane-located purine nucleotide transport proteins are key components for host exploitation by microsporidian intracellular parasites. PloS Pathogens 10: e1004547.

Huang Y., Zhen g S., Mei X., Yu B., Sun B., Li B., Wei J., Chen J., Li T., PAn G., Zhou Z., Li C. 2018: A secretory hexokinase plays an active role in the proliferation of Nosema bombycis. PeerJ. 6: e5658.

Krause D.C., Winkler H.H., Wood D.O. 1985: Cloning and expression of the Rickettsia prowazekii ADP/ATP translocator in Escherichia coli. Proc. Natl. Acad. Sci. U S A. 82: 3015-3019.

Li M., GaO Z., WANG Y., WANG H., Zhang S. 2014: Identification, expression and bioactivity of hexokinase in amphioxus: Insights into evolution of vertebrate hexokinase genes. Gene 535: 318-326.

Li Y., Wu Z., Pan G., He W., Zhang R., Hu J., Zhou Z. 2009: Identification of a novel spore wall protein (SWP26) from microsporidia Nosema bombycis. Int. J. Parasitol. 39: 391-398.
Pan G., Xu J., Li T., Xia Q., Liu S.L., Zhang G., Li S., Li C., Liu H., Yang L., Liu T., Zhang X., Wu Z., Fan W., Dang X., Xiang H., Tao M., Li Y., Hu J., Li Z., Lin L., Luo J., Geng L., Wang L., Long M., Wan Y., He N., Zhang Z., Lu C., Keeling P.J., Wang J., Xiang Z., Zhou Z. 2013: Comparative genomics of parasitic silkworm microsporidia reveal an association between genome expansion and host adaptation. BMC Genomics. 14: 186.

Reinke A.W., Balla K.M., Bennett E.J., Troemel E.R. 2017: Identification of microsporidia host-exposed proteins reveals a repertoire of rapidly evolving proteins. Nat. Commun. 8: 14023.

Taupin V., Méténier G., Vivarès C.P., Prensier G. 2006: An improved procedure for Percoll gradient separation of sporogonial stages in Encephalitozoon cuniculi (Microsporidia). Parasitol. Res. 99: 708-714.

Trentmann O., Horn M., Scheltinga A.C., Neuhaus H.E., HAFERKAMP I. 2007: Enlightening energy parasitism by analysis of an ATP/ADP transporter from chlamydiae. PLoS Biology 5: e231.

Tsaousis A.D., Kunji E.R., Goldberg A.V., Lucocq J.M., Hirt R.P., EMBLEY T.M. 2008: A novel route for ATP acquisition by the remnant mitochondria of Encephalitozoon cuniculi. Nature 453: 553-556.

VÁvRA J., Lukeš J. 2013: Microsporidia and 'the art of living together'. Adv. Parasitol. 82: 253-319.

Wilson J.E. 1995: Hexokinases. Rev. Physiol. Biochem. Pharmacol. 126: 65-198.

WiLson J.E. 2003: Isozymes of mammalian hexokinase: structure, subcellular localization and metabolic function. J. Exp. Biol. 206, 2049-2057.

Wiredu Boakye D., Jaroenlak P., Prachumwat A., Williams T.A., Bateman K.S., Itsathitphaisarn O., Sritunyalucksana K., Paszkiewicz K.H., Moore K.A., Stentiford G.D., Williams B. A. 2017: Decay of the glycolytic pathway and adaptation to intranuclear parasitism within Enterocytozoonidae microsporidia. Environ. Microbiol. 19: 2077-2089.

Wittner M., Weiss L.M. 1999: The Microsporidia and Microsporidiosis. American society for Microbiology Press, Washington, $553 \mathrm{pp}$.

Wolf A.J., Reyes C.N., Liang W., Becker C., Shimada K., Wheeler M.L., Cho H.C., Popescu N.I., Coggeshall K.M., Arditi M., Underhill D.M. 2016: Hexokinase is an innate immune receptor for the detection of bacterial peptidoglycan. Cell 166: 624-636.

Wu Z., Li Y., Pan G., Tan X., Hu J., Zhou Z., Xiang Z. 2008: Proteomic analysis of spore wall proteins and identification of two spore wall proteins from Nosema bombycis (Microsporidia). Proteomics. 8: 2447-2461 